Colder electronic samples and devices will enable searches for new physics in a large range of systems such as topological insulators, new collective electron-spin states, 2D materials, quantum Hall, exotic superconductors and quantum phase transitions. On the technology side lower electron temperatures may prove crucial for increasing the coherence times of solid-state qubits, reducing metrological uncertainty in resistance standards and increasing the sensitivity of detectors for astrophysics and cosmology.

\section{Why is it difficult?}

Readers familiar with the frontiers of low temperature physics may be asking themselves why this is such a problem; the cooling of bulk materials deep into the microkelvin regime has been possible for decades using the textbook technique of adiabatic demagnetisation of, say, chunks of high purity annealed copper forming a "demag stage" pre-cooled by a dilution refrigerator. Others may be wondering what is so difficult for nanoelectronic devices when laser-cooled atomic gases are regularly reported in the $\mathrm{pK}$ and $\mathrm{nK}$ regimes. The challenge becomes one of asking what cooling techniques are available (lasers are not, demagnetisation is)

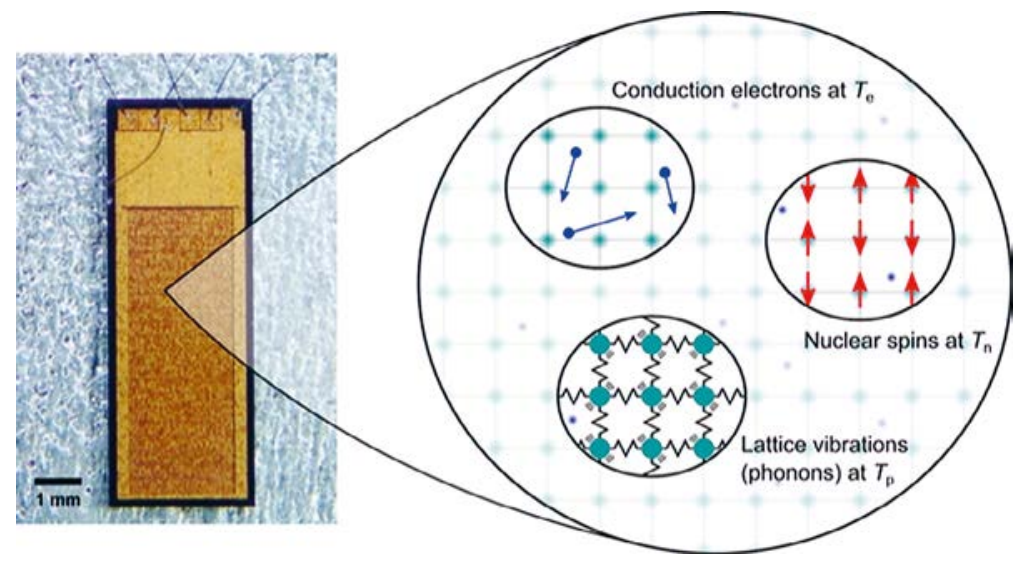

$\triangle$ FIG. 1: As the temperature of a material drops, it becomes increasingly easy to drive its internal subsystems out of thermal equilibrium. Left, an image of a Coulomb blockade thermometer [7], a device that measures its own internal electron temperature. Right, a sketch of the thermal subsystems inside its conducting parts. At millikelvin temperatures and below, the conduction electrons, crystal lattice and nuclear spins of the same material can all be at significantly different temperatures. It becomes harder to cool the conduction electrons with the refrigerator via contact with the lattice. In the device shown here the conduction electrons can instead be cooled via demagnetisation refrigeration of the nuclear spins in on-chip volumes of copper [8].

and what problems beset nanoscale devices in remaining cold (more susceptible to nuisance heating than bulk materials, but not so fragile as gas condensates).

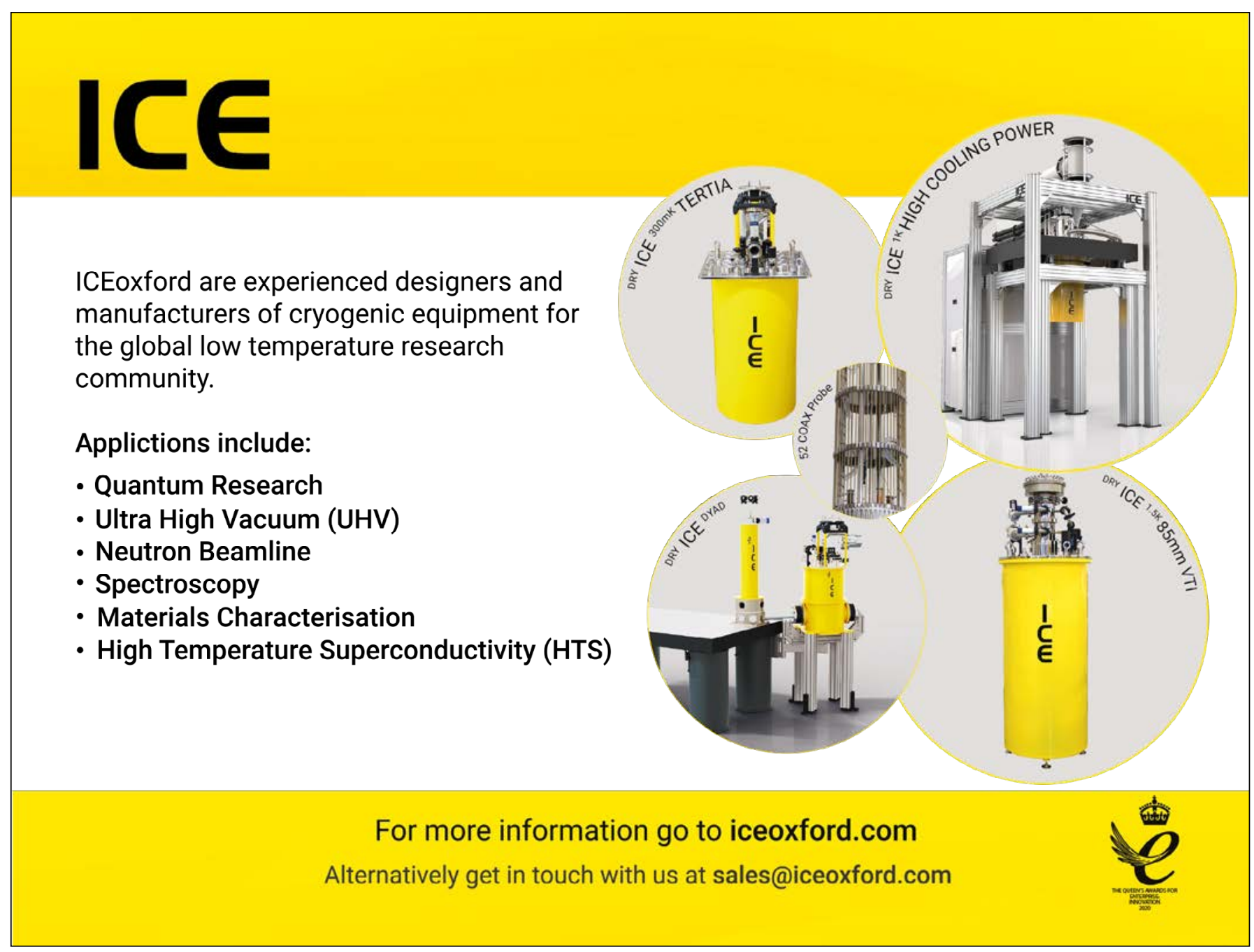


At low temperatures, the conduction electrons in a device decouple from the host lattice and, in turn, the cold chip substrate. See figure 1. At the same time the electron heat capacity reduces with temperature and exacerbates sensitivity to the many sources of parasitic heating such as radiation, electromagnetic noise and eddy currents. Ultimately the electron temperature is determined by the balance of cooling power vs parasitic heating.

Finally, one must measure the temperature of the electrons in the device, in equilibrium with the thermometer, to demonstrate that they are actually cold. This is no mean feat. The electrons are almost always at a temperature above that of the chip substrate which, in turn, will be hotter than the cold end of the refrigerator. Therefore, we choose a temperature-dependent property of the electrons themselves. Good examples are noise thermometers, the thermal properties of quantum dots, and Coulomb blockade thermometry.

\section{How do we do it and where are we now?}

Low temperature physicists are used to exploiting high field superconducting magnets for bulk demagnetisation cooling. See figure 2. Investigating how this technique might be applied to on-chip nano-fabricated devices was something of a natural progression. Earlier records were set by using ingenious ways of transferring the cold temperatures of large demag stages "off-chip" to the conduction electrons in a device. Typically, this is done by immersing the chips and measurements leads in cold liquid helium. The challenge is to transfer the cold while ensuring the device and leads are electrically isolated from the demag stage. Royal Holloway researchers employ these methods to cool 2D electron gases [4].

The Basel team developed a completely new approach to off-chip demagnetisation by cooling each measurement lead separately with its own mini demag stage and

VFIG. 2: Adiabatic demagnetisation cooling exploits the magnetocaloric effect where the temperature of certain materials can be changed by the application of a magnetic field. Paramagnetic indium or copper (as shown here) is pre-cooled under high magnetic field using a dilution refrigerator and superconducting solenoid. The key to the technique is to reduce the field whilst protecting the material from external heat leaks in order to follow the constant entropy adiabat as closely as possible. This can result in significant cooling of the material below the base temperature of the refrigerator.

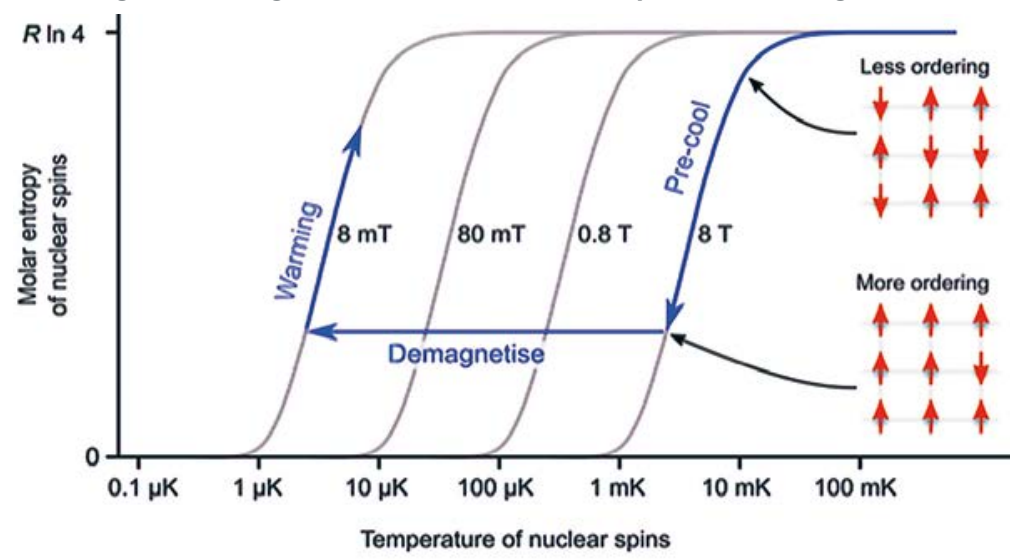

transferring this cooling via the chip's electrical contacts. This versatile and adaptable technique has been demonstrated in combination with modern cryogen-free refrigerators and successfully cooled 16 demagnetised measurement wires to fractions of a $\mathrm{mK}$ [5].

Meanwhile, VTT and Lancaster decided to deposit demagnetisation material directly on to chips and investigate whether it would cool. Serendipitously it did. The key was to add the material to a device that was also an accurate thermometer of electron temperature. Coulomb blockade thermometers made by Mika Prunnila's team at VTT had already been shown to work down to $4 \mathrm{mK}$ in an immersion cooling experiment [7]. Coating the conducting parts with copper provided a new on-chip cooling method. The total amount of copper was approximately 4 $\mu \mathrm{mol}, 5$ or 6 orders of magnitude less than would be used in a traditional demag stage, but the thermal link between the copper and the device was essentially perfect. Starting from the base temperature of a cryogen-free dilution refrigerator and a magnetic field of $5 \mathrm{~T}$, electrons were cooled from $9 \mathrm{mK}$ to $5 \mathrm{mK}$ [8]. The most surprising result was how long the tiny amount of copper stayed cold: just below $5 \mathrm{mK}$ for up to 1000 seconds. Measurements made using a colder dilution refrigerator were able to reach $1.1 \mathrm{mK}$.

Combining both off-chip and on-chip techniques, as demonstrated in Basel [6], and also replacing copper with indium, the team led by Attila Geresdi, formerly at Delft and now at Chalmers, made a breakthrough by cooling the electrons in a Coulomb blockade thermometer below $1 \mathrm{mK}$ (see Fig. 3). They realised that indium has a significantly larger cooling power than copper and this, combined with external cooling of the leads, allowed them to cool the electrons to $420 \mu \mathrm{K}$ and keep them below $700 \mu \mathrm{K}$ for more than 85 hours [9]. The base temperatures achievable with indium will ultimately be limited by its magnetic properties at very low temperatures and fields. Consequently, copper is capable of producing lower temperatures, but indium certainly has advantages above $400 \mu \mathrm{K}$.

Right now at Basel an experimental campaign is underway that combines everything we have learned so far: off-chip, on-chip and the best choices of thermometry and materials for each stage. Preliminary results indicate base temperatures around $300 \mu \mathrm{K}$ and days-long sub-mK hold times.

\section{What next?}

As well as setting new records, we have demonstrated that it is possible to cool electrons in on-chip nanofabricated devices well below temperatures currently accessible on commercial refrigerators. New tools lead to new physics, and one of the next challenges is identifying which of the new techniques is best suited for particular on-chip applications and investigations beyond those mentioned here. A key aspect of the European Microkelvin Platform 


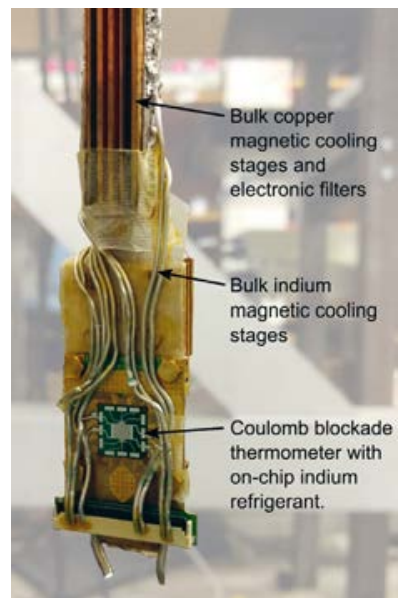

4 FIG. 3: The cold stage of an onchip demagnetisation experiment at TU Delft [9]. The top of this setup attaches to the coldest part of a dilution refrigerator, which is at a temperature around $\mathbf{1 0} \mathrm{mK}$. Bulk copper and indium demagnetisation stages cool the incoming connections to the sample. 0 ther sources of external heating are reduced by careful electronic filtering and shielding. Finally, microscopic amounts of indium on the sample itself are able to cool the on-chip electrons to temperatures close to $400 \mu \mathrm{K}$. Photograph by Matthew Sarsby, TU Delft.

is to bring innovation out of the laboratory and into the marketplace. Ultimately, we would like to see off- and onchip cooling become easily accessible and cost-effective, for example as an add-on option to commercial cryogen-free dilution refrigerators.

\section{About the Authors}

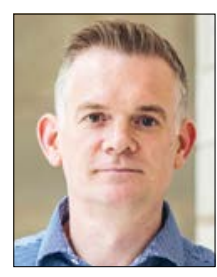

Richard Haley is a Professor of Low Temperature Physics at Lancaster University. He has interests in cooling and performing sensitive measurements on a range of systems including nano-fabricated structures and superfluid helium-3 condensates.

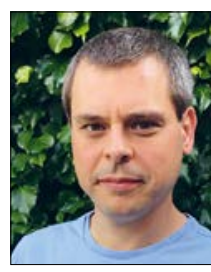

Jonathan Prance is a Reader in Physics and the Director of IsoLab at Lancaster University. He studies the properties of nanoelectronic structures and devices at extreme low temperatures, including quantum dots, 2D materials and superconducting circuits.

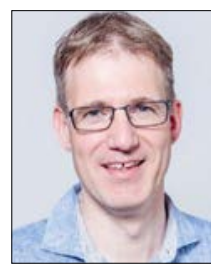

DominikZumbühl is a Professor of Physics at the University of Basel working on quantum transport in nanostructures studying quantum coherence, spins and interactions at low temperatures. He is also the Director of NCCR SPIN, the Swiss National Program developing spin qubits in $\mathrm{Si}$ and $\mathrm{Ge}$.

\section{References}

[1] Jones et al., J. Low Temp. Phys. 201, 772 (2020)

[2] Pan et al., Phys. Rev. Lett. 83, 3530 (1999)

[3] https://emplatform.eu

[4] Beauchamp et al., Appl. Phys. Lett. 117, 162104 (2020)

[5] Palma et al., Rev. Sci. Instr. 88, 043902 (2017)

[6] Palma et al., Appl. Phys. Lett. 111, 253105 (2017)

[7] Bradley et al., Nat. Commun. 7, 10455 (2016)

[8] Bradley et al., Sci. Rep. 7, 45566 (2017)

[9] Sarsby et al., Nat. Commun. 11, 1492 (2020)

\section{THYR $\triangle \mathrm{CONT}$

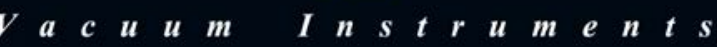

\section{Faster Vacuum Process Integration - via USB Transducers}

\begin{abstract}
A new type of combi-transducer with USB connection complements the product portfolio of Thyracont Vacuum Instruments. High accuracies and wide measuring ranges have become standard at Thyracont. Now a vacuum transducer with a brand-new special feature in the form of a USB-C connection has been developed. This communication interface increases flexibility and enables quick and easy interconnection to other portable devices via USB-C. Such compatibility allows a plug \& play connection to all common computers or Android smartphones in order to display the measured vacuum precisely and quickly during process monitoring.
\end{abstract}

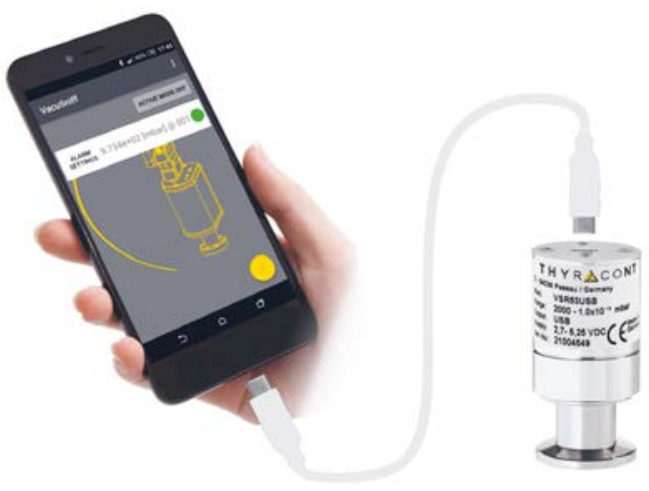

There is no need for a separate power supply, as the reduced power consumption of the device is covered by all standard computers and smartphones. The exact digital readjustment to atmospheric or zero pressure, as well as many other functions which support the monitoring, can be carried out intuitively using our VacuGraphTM software, which is available for various operating systems. In addition, the software is supplemented by the Android app VacuSniff to support visualization of measurements on smartphones and tablets. The combination of mobile devices and USB-C allows to put sensors into operation while the vacuum process is running and can this way upgrade the process with improved monitoring.

For more information, contact: Thyracont Vacuum Instrument https://thyracont-vacuum.com/en +49851 95986-0 